Article

\title{
Cryptococcus neoformans Capsular GXM Conformation and Epitope Presentation: A Molecular Modelling Study
}

\author{
Michelle M. Kuttel ${ }^{1, * \mathbb{C}}$, Arturo Casadevall ${ }^{2} \mathbb{C}$ and Stefan Oscarson ${ }^{3}$ \\ 1 Department of Computer Science, University of Cape Town, Cape Town 7701, South Africa \\ 2 Department of Molecular Microbiology and Immunology, Johns Hopkins Bloomberg School of Public Health, \\ Johns Hopkins University, 615 N Wolfe St Room E5132, Baltimore, MD 21205, USA; acasade1@jhu.edu \\ 3 Centre for Synthesis and Chemical Biology, University College Dublin, Belfield, Dublin 4, Ireland; \\ stefan.oscarson@ucd.ie \\ * Correspondence: mkuttel@cs.uct.ac.za; Tel.: +27-21-6505107
}

Received: 3 May 2020; Accepted: 4 June 2020; Published: 7 June 2020

\begin{abstract}
The pathogenic encapsulated Cryptococcus neoformans fungus causes serious disease in immunosuppressed hosts. The capsule, a key virulence factor, consists primarily of the glucuronoxylomannan polysaccharide (GXM) that varies in composition according to serotype. While GXM is a potential vaccine target, vaccine development has been confounded by the existence of epitopes that elicit non-protective antibodies. Although there is evidence for protective antibodies binding conformational epitopes, the secondary structure of GXM remains an unsolved problem. Here an array of molecular dynamics simulations reveal that the GXM mannan backbone is consistently extended and relatively inflexible in both $C$. neoformans serotypes A and D. Backbone substitution does not alter the secondary structure, but rather adds structural motifs: $\beta$ DGlcA and $\beta$ DXyl side chains decorate the mannan backbone in two hydrophillic fringes, with mannose-6-O-acetylation forming a hydrophobic ridge between them. This work provides mechanistic rationales for clinical observations-the importance of O-acetylation for antibody binding; the lack of binding of protective antibodies to short GXM fragments; the existence of epitopes that elicit non-protective antibodies; and the self-aggregation of GXM chains-indicating that molecular modelling can play a role in the rational design of conjugate vaccines.
\end{abstract}

Keywords: Cryptococcus neoformans; conjugate vaccines; GXM; conformation; capsular polysaccharide; epitope; carbohydrate antigen; molecular modelling

\section{Introduction}

Cryptococcus neoformans is an opportunistic invasive fungus that causes serious disease (typically meningitis) in immunosuppressed hosts [1]. Cryptococcus species are encapsulated fungi, with a thick, hydrophillic capsule that covers the cell wall surface, forms a protective biofilm and is the chief virulence factor [2]. The capsule is primarily composed of a glucuronoxylomannan polysaccharide (GXM, around $90 \%$ ); further minor components are a galactomannan polysaccharide (GXMGal, 10\%) and mannoproteins $(<1 \%)$ [3]. C. neoformans is currently classified into two varieties known as neoformans and grubii [4]. There are three serotypes of $C$. neoformans, referred to as serotype D (Cryptococcus neoformans var. neoformans), serotype A (Cryptococcus neoformans var. grubii) and serotype AD (hybrid). Globally, serotypes A and D are responsible for the vast majority of infections in humans [4]. Serotypes B and C are now classified as a separate species, Cryptococcus gattii. However, genomic analysis suggests that both the $C$. neoformans and $C$. gattii groupings include various species and these are currently viewed as species complexes [5]. 
The serotype classifications for Cryptococcus are on the basis of antigenic differences arising from structural variations in GXM [6]. Light scattering and hydronamic studies suggest that GXM is a branched polymer comprising an $\alpha(1 \rightarrow 3)$ mannose backbone substituted with $\beta(\rightarrow 2)$ glucuronic acid every third mannose, along with variable $\beta(1 \rightarrow 2)$ and $\beta(1 \rightarrow 4)$ xylose side chains $[7,8]$. Some mannose residues are 6-O-acetylated (up to $60 \%$ ) and O-acetylation plays a key role immune recognition of GXM [9]. The GXM polysaccharide has considerable heterogeneity [10]: in contrast to bacterial polysaccharides that have a single oligosaccharide repeat unit (RU), GXM has at least six repeat motifs (called triads), and a single molecule of GXM may include different motif combinations [11]. For each Cryptococcus serotype, the GXM structure has a major triad and one or more minor triads. For serotype $\mathrm{A}$, the dominant triad is a six-residue RU with two $\beta(1 \rightarrow 2)$ xylose side chains (triad M2), whereas serotype $\mathrm{D}$ has a dominant five-residue RU with one $\beta(1 \rightarrow 2)$ xylose side chain (triad M1), as shown in Figure 1. Serotypes A and D have an estimated average of two acetates per triad [12].

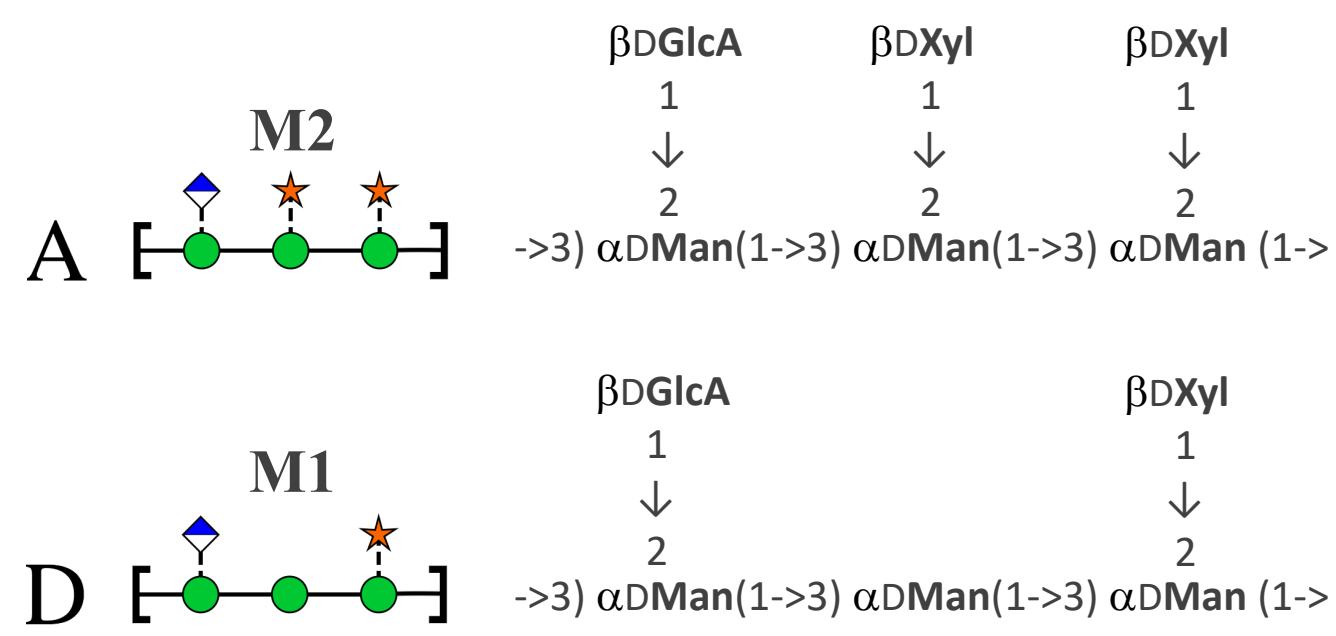

Figure 1. The predominant GXM repeat unit motif (triad) in C. neoformans serotypes. Serotype A (Cryptococcus neoformans var. grubii, top) has predominantly the M2 triad and serotype D (Cryptococcus neoformans var. neoformans, bottom) the M1 triad [11]. The RUs are shown on the left with the SNFG symbols $[13,14]$.

GXM is a promising antigen in vaccine development since a polysaccharide-protein conjugate vaccine could potentially provide effective protection [15]. However, there have been complications following this route, because the GXM polysaccharide is poorly immunogenic and has been found to elicit both protective and non-protective and even deleterious antibodies in humans [16]. This led to the hypothesis that GXM contains some epitopes eliciting protective antibodies and other epitopes eliciting non-protective antibodies, with a non-protective antibody response impeding the formation of protective antibodies [17]. There is evidence that some epitopes that elicit protective antibodies are conformational [18] and O-acetylation is known to be critical for binding of protective antibodies [19,20]. One approach to identifying protective GXM epitopes is the creation of a library of synthetic oligosaccharides which are then conjugated to protein carriers and tested in mice to identify immunogenic motifs that are potential vaccine candidates [18,21]. In the first stages of this strategy, none of the smaller synthesised molecules (from a tetra to a heptasaccharide) were recognised by protective antibodies; a synthetic heptasaccharide representing the GXM serogroup A triad (M2) conjugated to human serum album raised a non-protective immune response in mice [17]. However, recently a synthetic decasaccharide was shown to bind to a number of protective antibodies and is therefore a promising vaccine candidate [18]. 
It has been hypothesised that differences in the degree of side-chain substitution and O-acetylation give rise to changes in the secondary structure of the GXM polysaccharide that directly affect $\mathrm{Ab}$ binding and hence antigenicity [9]. However, despite postulates of the existence of conformational epitopes of GXM $[9,11,17]$, the secondary structure of GXM is still unknown and is recognised as a gap in the understanding of $C$. neoformans capsule [20]. In addition, the mechanisms through which the GXM polysaccharides (and other capsule molecules) assemble into a capsule remain largely undiscovered [20], although there are indications that GXM molecules self-aggregate, possibly mediated by divalent cations [22-24]. In the absence of experimental evidence on secondary structure (which is extremely challenging to obtain for flexible polysaccharides), molecular modelling has been demonstrated to provide insights into molecular conformation, biophysical dynamics and interactions that can usefully inform vaccine development [25].

In this work we employ molecular dynamics simulations on an array of oligosaccharides (Figure 2) to establish the conformation of GXM in serotype A and D, aiming to investigate the following questions.

1. What is the secondary structure or conformation of GXM?

2. Does the differing xylose substitution pattern in serotypes A and D alter the GXM conformation?

3. Does 6-O-acetylation on the mannose backbone alter the conformation of serotype A?

4. Do shorter strands with the M2 triad have the same conformational epitope as the GXM serotype A polysaccharide?

5. What is mechanism of GXM self-aggregation?

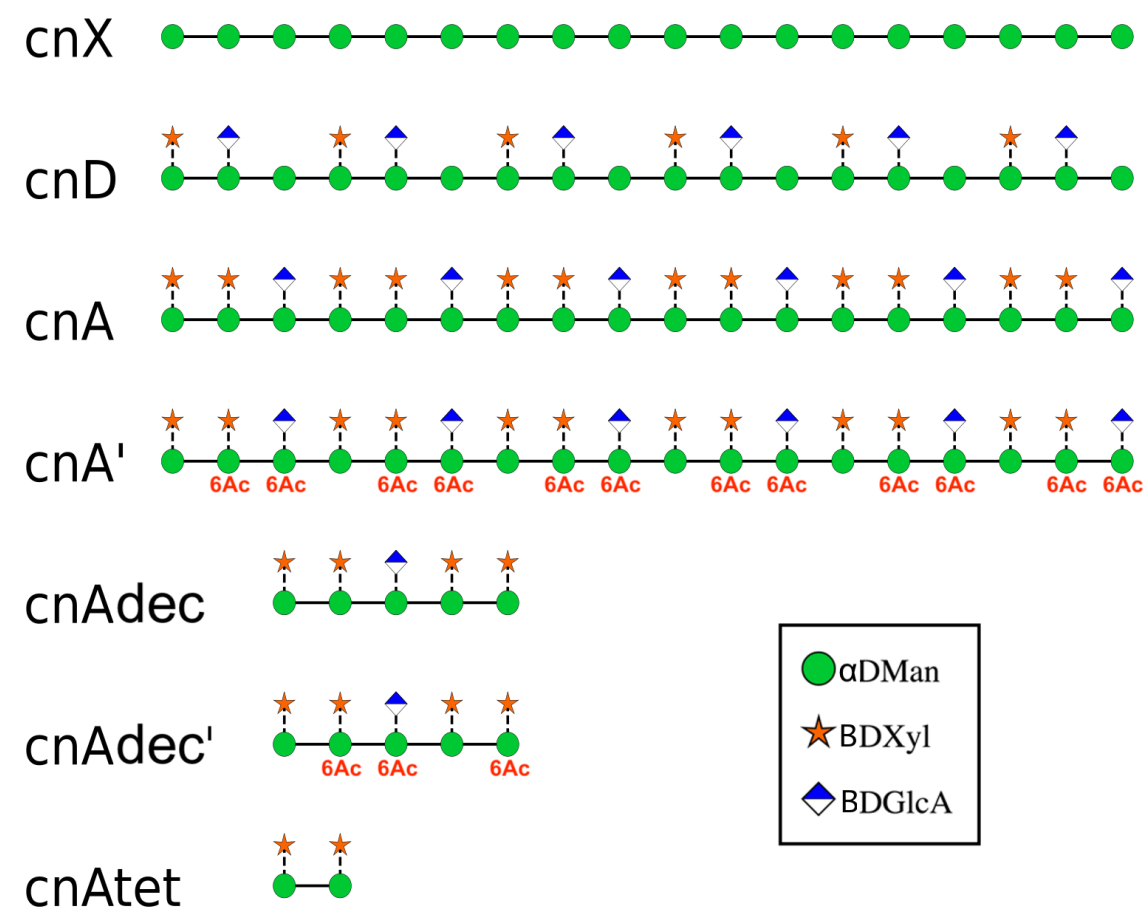

Figure 2. The array of GXM oligosaccharides simulated in this work, shown with the SNFG symbols $[13,14]$ for the sugar residues. Six RUs of an unsubstituted backbone (cnX); the main repeat motifs in serogroup D ( $\mathrm{cnD})$ serogroup A ( $\mathrm{cnA}$ ) and 6-O-acetylated serogroup A ( $\left.\mathrm{cnA}^{\prime}\right)$ were run in separate simulations; and three shorter strands of serogroup A GXM: a decasaccharide (cnAdec), a 6-O-acetylated decasaccharide ( $\left.\mathrm{cnAdec}^{\prime}\right)$ and a tetrasaccharide (cnAtet). 
To investigate the effects of the side chain substitutions on GXM conformation, we ran molecular dynamics simulations in an aqueous solution of 6 RUs of an unsubstituted $\alpha(1 \rightarrow 3)$ mannose backbone (hereafter termed $\mathrm{cnX}$ ), for comparison purposes, and simplified 6-RU chains of serogroup A (cnA) and serogroup $\mathrm{D}(\mathrm{cnD})$. For the simplified strands, the saccharide chains comprised simple repeats of the dominant triad motif for the serogroup (as shown in Figure 2). To investigate the effect of $\mathrm{O}$-acetylation on GXM, we simulated serogroup A with 6-O-acetylation on the first and third mannose in the RU $\left(\mathrm{cnA}^{\prime}\right)$. This pattern was chosen to match that in the synthetic decasaccharide [18] and is in accordance with the estimated average of two acetates per triad [12]. Then, to investigate the effect of chain length on conformation, we simulated a tetrasaccharide (cnAtet) as well as the decasaccharide representing promising vaccine candidate, the latter both being 6-O-acetylated (cnAdec') and de-O-acetylated (cnAdec). Finally, to look at the possibility of GXM self-aggregation, we ran a simulation of $\mathrm{cnA}$ and $\mathrm{cnD}$ together, to allow for possible spontaneous association of the chains. The trajectories of these multiple simulations were then analysed and compared, as discussed below.

\section{Results}

We first compare the effects of substitution of the GXM mannan backbone on chain flexibility and proceed to a comparison of the secondary structure in $\mathrm{cnD}, \mathrm{cnA}$ and $\mathrm{cnA}^{\prime}$. We then contrast the conformation of shorter fragments of serotype A with the 6-RU strands. Finally, we look at the potential for GXM chain self-aggregation.

\subsection{Comparison of GXM Chain Flexibility}

A simple measure of chain flexibility is the fluctuation in end-to-end distance, $r$, for a molecule over the course of a simulation. Figure 3 shows the $r$ time series and corresponding histograms for (a) $c n X,(b) c n D,(c) c n A$ and (d) $c^{\prime} A^{\prime}$. Here we define $r$ for all 6-RU GXM chains as the distance from O3 in the second linkage in the mannose chain to the $\mathrm{O} 3$ in the second last linkage, thereby excluding the more flexible two terminal residues on either end of the chain (labeled on the cnA molecule in Figure 3, right). The $\alpha(1 \rightarrow 3)$ mannan chain is generally extended: the distributions of $r$ (Figure 3 right column) are tight and skewed to the right (larger $r$ values), with all the four saccharides having a narrow peak at the median chain length of $54 \AA$. The mannan backbone is thus remarkably inflexible, as there are no short $r$ distances indicating bends that bring the ends of the chain into close proximity; although transient "elbow" bends do occur occasionally, they do not persist. Further, comparison of the $r$ plots shows a trend of decreasing chain flexibility with increasing chain substitution: the unsubstituted $\mathrm{cn} X$ backbone is the most flexible with the broadest spread in $r(\sigma=2.8)$; this flexibility is decreased in cnA $(\sigma=2.3)$ and the most substituted $\mathrm{cnA}^{\prime}$ is markedly the least flexible, with the narrowest range of $r$ $(\sigma=1.6)$.

This trend of reduced flexibility with increased backbone substitution is further illustrated by the decreasing range of rotation in the backbone mannose $\alpha(1 \rightarrow 3)$ linkages, shown in the heat maps in Figure 4a. Although the dominant orientation of this linkage is the same in $\mathrm{cnX}, \mathrm{cnD}, \mathrm{cnA}$ and $\mathrm{cnA}^{\prime}$ (indicating the same backbone conformation in all 6-RU GXM molecules), the range of motion is greatest in $\mathrm{cnX}$ and decreases in the order $\mathrm{cnD}, \mathrm{cnA}$ and $\mathrm{cnA}^{\prime}$. 
(a)
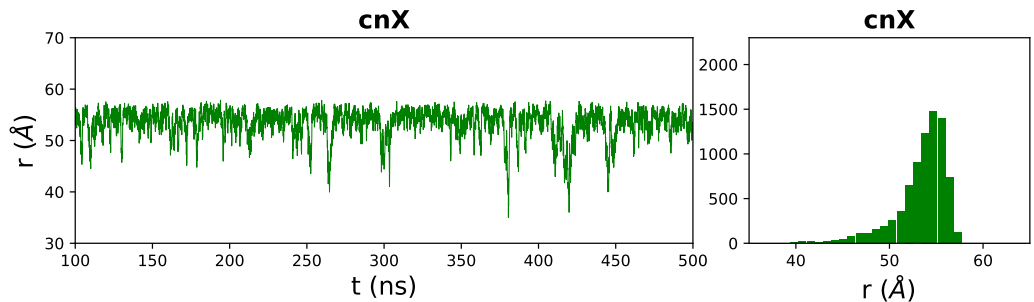

(b)
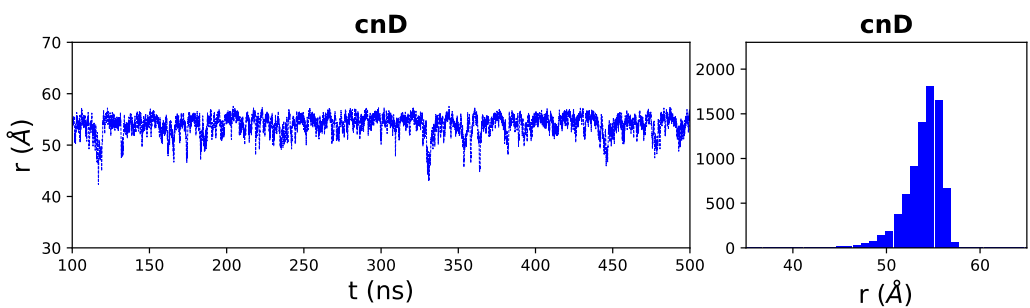

(c)
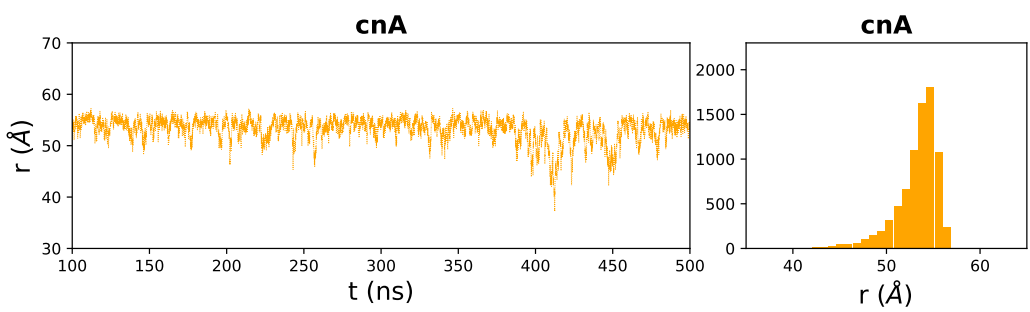

(d)
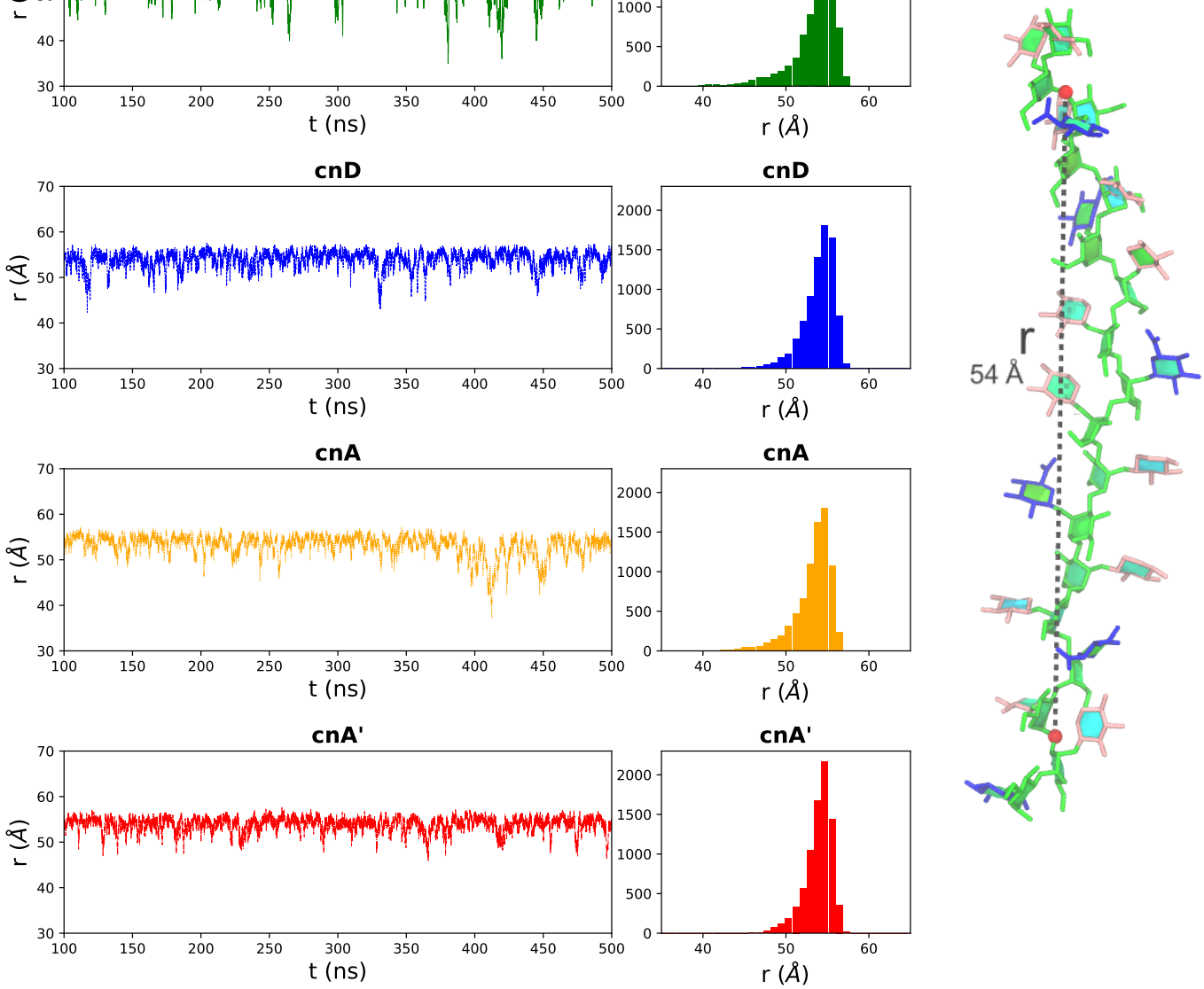

Figure 3. End-to-end distance, $r$, time series and corresponding histograms for the $(\mathbf{a}) \mathrm{cn} X,(\mathbf{b}) \mathrm{cnD}$, (c) $\mathrm{cnA}$ and (d) $\mathrm{cnA}^{\prime}$ GXM chains. The first $100 \mathrm{~ns}$ of simulation are considered equilibration and are not shown. Here $r$ is defined to exclude the two terminal residues on either end of the chain, as the distance from $\mathrm{O} 3$ in the second linkage to $\mathrm{O} 3$ in the 16th linkage in the 18-mannose backbone-labelled for $\mathrm{cnA}$ in the image on the right. The residues and substitutions are coloured as follows: $\alpha$ DMan-green; $\beta$ DGlcA-blue; $\beta$ DXyl-pink; and 6-OAc-red.

The orientation of the $\beta$ DGlcA $(1 \rightarrow 2) \alpha \operatorname{DMan}$ (Figure $4 \mathrm{~b})$ and $\beta \mathrm{DXyl}(1 \rightarrow 2) \alpha \mathrm{DMan}$ (Figure $4 \mathrm{c}$ ) side chains does not vary greatly across $\mathrm{cnD}, \mathrm{cnA}$ and $\mathrm{cnA}$. However, the side chains are considerably more mobile than the backbone (compare the narrower extent of the heat maps in Figure 4a). Both side chains are predominantly in a "face-on" orientation to the mannan backbone (insets i in Figure 4b,c), where the plane of the ring and hence hydrophillic ring hydroxyls are aligned parallel to the mannan backbone. This orientation is the global energy minimum for the $\beta$ DGlcA $(1 \rightarrow 2) \alpha \mathrm{DMan}$ on the vacuum PMF (Appendix A, Figure A1). The side chains also have a minor "edge-on" conformation (insets ii in Figure $4 b, c$ ), where the plane of the ring and hence hydrophillic ring hydroxyls are aligned perpendicular to the mannan backbone. This orientation is a local energy minimum for

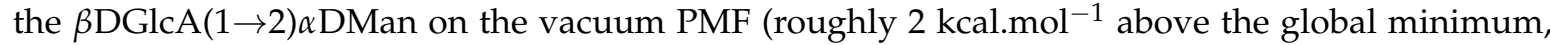
Figure A1). Note that a comparison of $\mathrm{cnA}$ and $\mathrm{cnA}^{\prime}$ in Figure 4 shows that $6-\mathrm{O}-\mathrm{Ac}$ substitution does not significantly affect orientation of the $\beta$ DGlcA and $\beta$ DXyl side chains. 
(a)
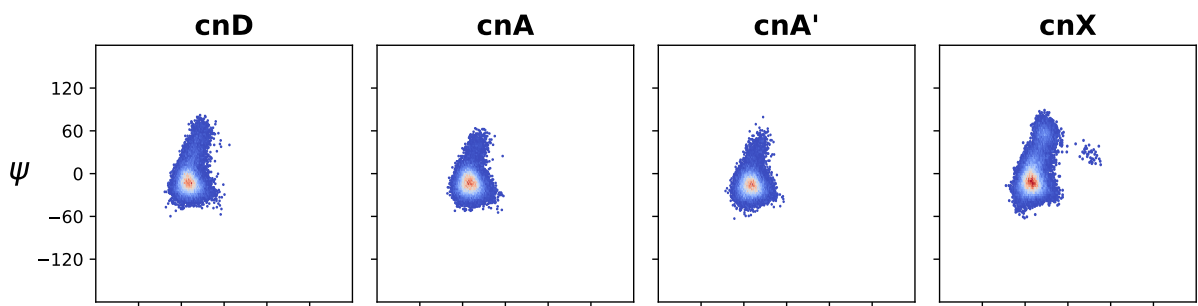

(b)
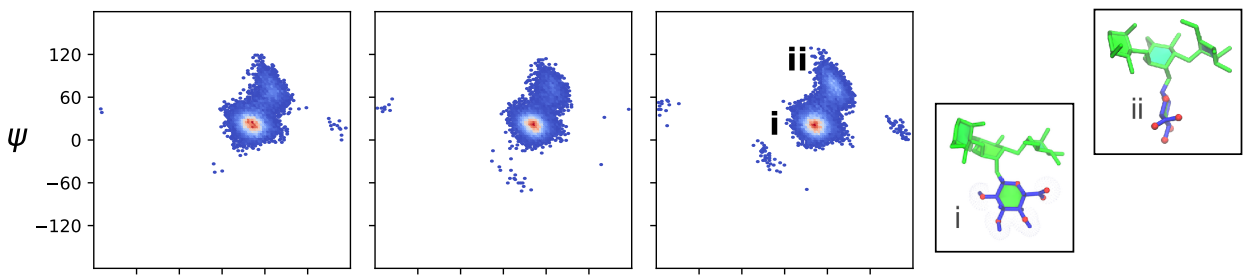

(c)
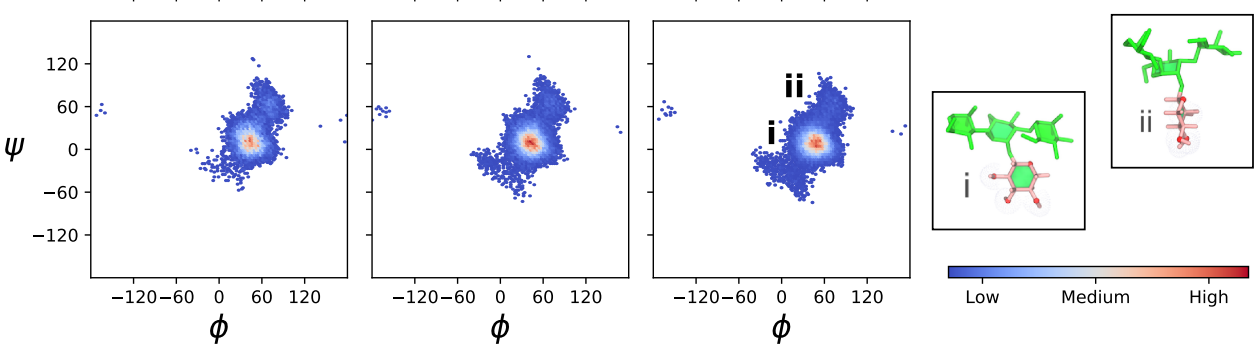

Figure 4. Heat maps of the glycosidic linkage orientation distribution over the last $400 \mathrm{~ns}$ of simulation in the two central RUs (RU3 and RU4) in the GXM chains. Row (a) shows the backbone $\alpha \operatorname{DMan}(1 \rightarrow 3) \alpha$ DMan linkage, row $(\mathbf{b})$ the $\beta$ DGlcA $(1 \rightarrow 2) \alpha$ DMan side chain linkage and row $(\mathbf{c})$ the $\beta \operatorname{DXyl}(1 \rightarrow 2) \alpha \mathrm{DMan}$ side chain linkage. Glycosidic linkage orientations are shown as rotations of the linkage dihedral angles $\phi$ and $\psi$. The insets in row (b,c) show the major face-on (i) and minor edge-on (ii) orientations of the $\beta(1 \rightarrow 2)$-linked side chains relative to the mannan backbone. The residues and substitutions are coloured as follows: $\alpha$ DMan—green; $\beta$ DGlcA—blue; $\beta$ DXyl—pink; and 6-OAc-red.

\subsection{Comparison of GXM Chain Conformations and Binding Surfaces}

Although the flexibility of the GMX molecules decreases with increasing substitution, the backbone conformation does not change significantly. Conformational clustering reveals that all the GXM chains have a strongly dominant molecular conformation, shown for each of the GXM molecules in Figure 5. For all molecules, this dominant conformation corresponds to the peak in the $r$ histogram (see Figure A3). In addition, the prevalence of this primary conformation increases in the order $\operatorname{cnX}(51 \%)<\operatorname{cnD}(55 \%)<\operatorname{cnA}(57 \%)<\mathrm{cnA}^{\prime}(69 \%)$ - A further indication that in GXM the chain flexibility decreases with increasing chain substitution. In all conformations, the backbone is extended, as is most apparent in the unsubstituted cnX conformation (Figure 5a), but is also clear for $\mathrm{cnD}$ (Figure $5 \mathrm{~b}$ ), cnA (Figure 5c) and $\mathrm{cnA}^{\prime}$ (Figure $5 \mathrm{~d}$ ). The backbone twists dynamically from flatter ribbon-like conformations to extended helical conformations, but its behaviour is relatively unaffected by the presence or absence of side-chain substitutions or O-acetylation. The sole effect of increasing substitution on the backbone is to constrain rotation about the backbone linkages. Therefore, the theory that the conformation of the backbone is changed with altered substitution patterns [9] is not supported by our results. 


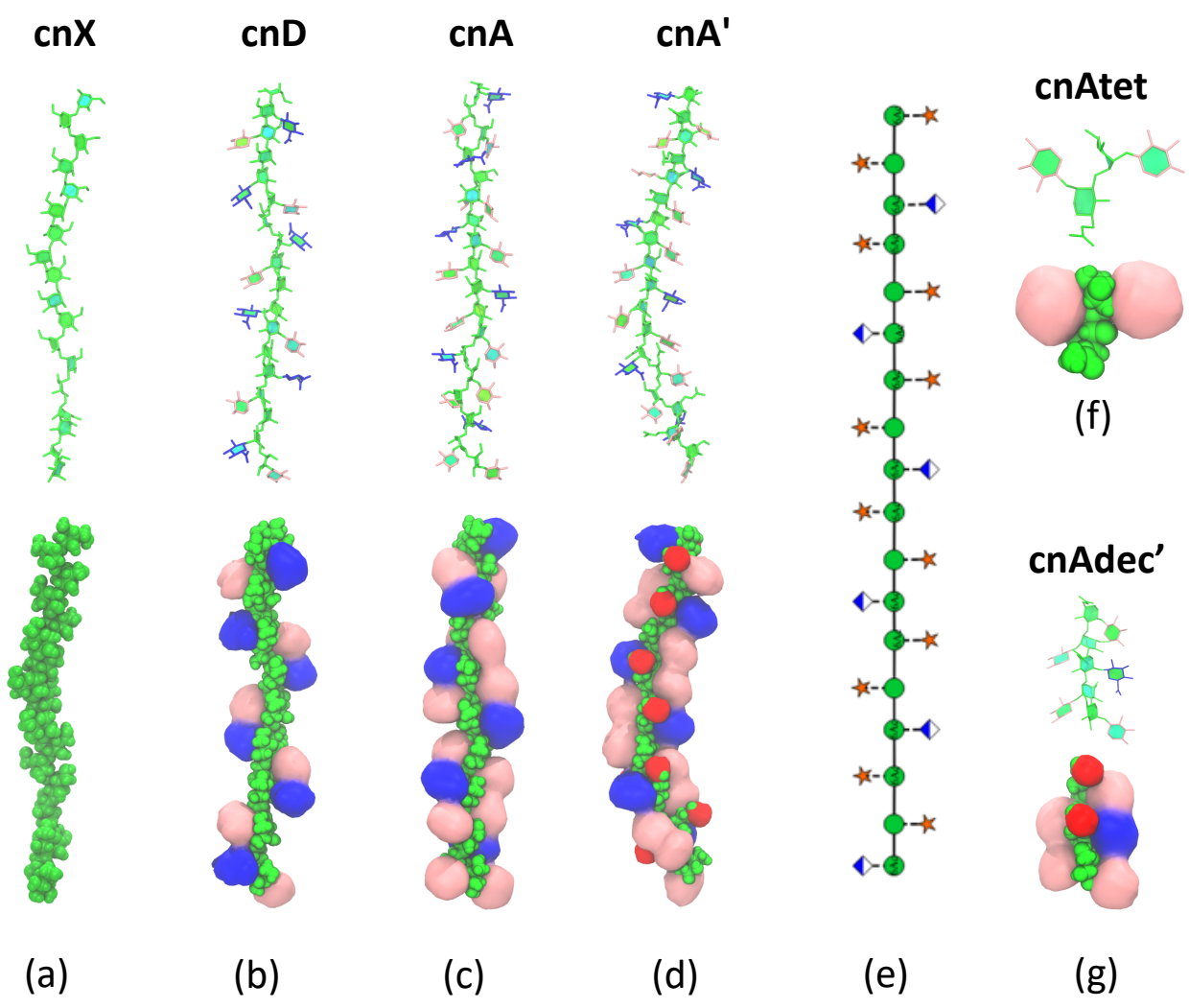

Figure 5. Dominant conformations of the GXM molecules shown with two representations in the VDM package [26] - the PaperChain visualisation [27] to highlight the conformation of the backbone and space-filling representations to highlight the exposed binding surface. Representative conformations are shown for (a) the unsubstituted mannan backbone cnX; (b) cnD; (c) cnA; (d) and cnA' (6-O-acetylated). A schematic for the arrangement of the side chain substitutions is shown in (e) using the SNFG symbols for the sugar residues $[13,14]$. Representative conformations of the chain fragments are shown for the tetrasaccahride (f) cnAtet and the 6-O-acetylated decasaccharide (g) cnAdec'. The chain substitutions in all representations are coloured as follows: $\alpha$ DMan-green; $\beta$ DGlcA—blue; $\beta$ DXyl—pink and 6-OAc-red.

The $\beta$ DGlcA and $\beta D X y l$ side chains are arranged along the backbone in two hydrophillic fringes, primarily in the "face-on" orientation with the ring hydroxyl groups aligned in the same plane as the mannan backbone. Side chains in the same fringe are in close proximity to each other and interact with each other via a dynamic network of hydrogen bonds, shifting closer and further away within a restricted range of $10 \AA$ (see Appendix A, Figure A4). However, it is alternating (not neighbouring) side chains in the primary structure that are in close proximity in the same fringe in the secondary structure-as shown in the schematic in Figure (Figure 5e). This observation is by no means clear from simple inspection of the primary structure of the GXM serotypes.

The side chain fringes are exposed and thus potentially form $\mathrm{Ab}$ binding surfaces-this is seen more clearly in the space filling structure representations in the bottom row of Figure 5. The molecular surface differs considerably across the serogroups. The fringes in the $\mathrm{cnD}$ structure (Figure $5 \mathrm{~b}$ ) comprise repeated patches of $\beta$ DGlcA - $\beta$ DXyl side chain pairs in close proximity, with gaps between them where the mannose backbone is exposed. In contrast, in cnA (Figure $5 \mathrm{c}$ ) the additional $\beta \operatorname{DXyl}(1 \rightarrow 2)$ side chain in each trimer creates a very different binding surface, forming $\beta$ DGlcA- $\beta$ DXyl- $\beta$ DXyl patches in a continuous band in each side chain fringe. Further, in $\mathrm{cnA}^{\prime}$ (Figure $5 \mathrm{~d}$ ) the 6-O-acetyl substitution forms a highly exposed hydrophobic ridge between the side chain fringes, which further conceals the mannan backbone. 
The shorter chain fragments do not differ in conformation from the larger oligosaccharides. For the serogroup A tetrasaccharide cnAtet (Figure 5f) and the two decasaccharides cnAdec and cnAdec' (Figure 5g), the side chains and the backbone glycosidic linkages have the same orientations as in the 6-RU saccharides (see Appendix A, Figure A2) - there is no conformational change in these shorter strands as compared to the longer chains.

\subsection{GXM Self-Aggregation}

Physical chemical studies have provided evidence that GXM molecules have the capacity for self-aggregation, but the mechanism for this effect is unknown [22]. Our simulation of $\mathrm{cnD}$ and $\mathrm{cnA}^{\prime}$ together shows evidence for self-aggregation, after $300 \mathrm{~ns}$ of equilibration of the system where the chains diffused in the water box. After this, there were repeated instances of prolonged $(\approx 50 \mathrm{~ns})$ spontaneous inter-locking of the two chains. We found two modes of interaction between GXM chains to occur. Firstly, a parallel head-to-tail orientation, where the side chains of one GXM molecule interacted with the backbone mannan of the neighbouring molecule. Figure 6a shows an example of this interaction. Here the $\mathrm{cnA}^{\prime}$ is rotated around the backbone axis by $90^{\circ}$ relative to $\mathrm{cnD}$, to bring the side chains of the $\mathrm{cnA}^{\prime}$ molecule into contact with the mannan backbone in $\mathrm{cnD}$ (illustrated in the cross-sectional schematic on the right of Figure 6a). The interacting side chains in both strands are in the major "face-on" orientation.

(a)

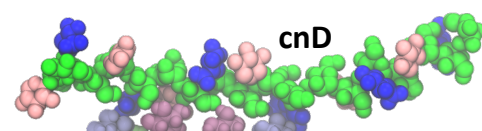

)
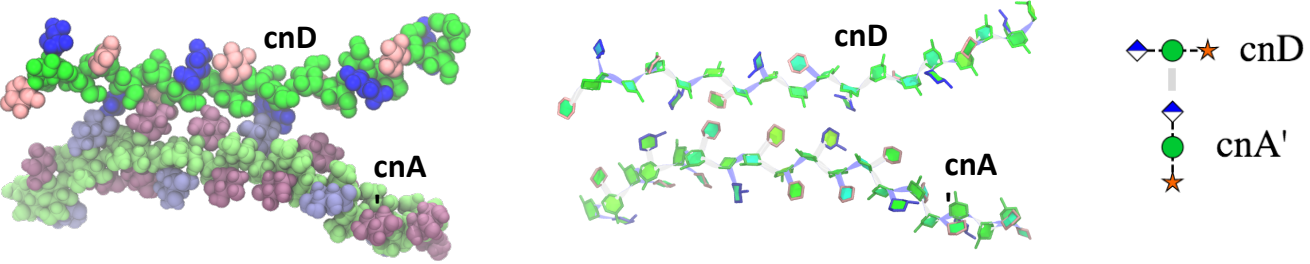

(b)
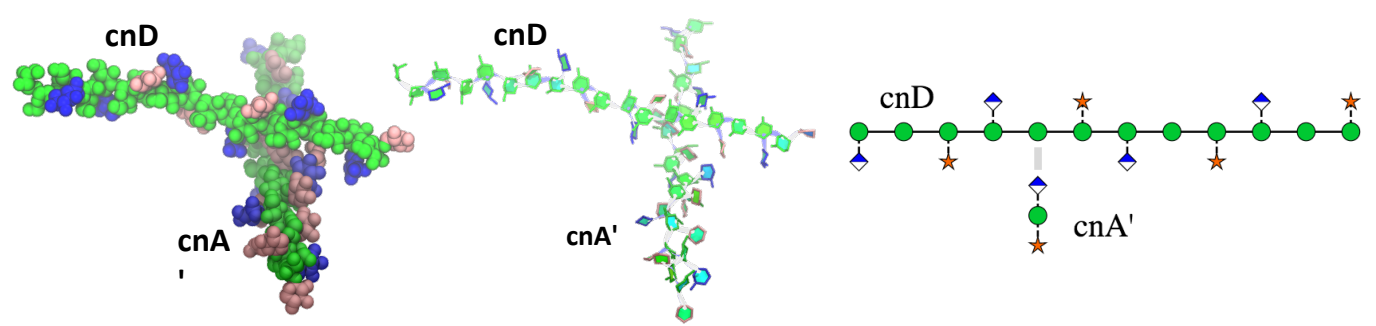

Figure 6. Examples of self-aggregation interactions between 6-RU strands of cnD and cnA in (a) parallel and (b) orthogonal arrangements. Molecules are depicted using the VMD [26] van der Waals representation (left); and the VMD PaperChain and Twister representations (middle) and a schematic (right). The saccharide residues are coloured as follows: $\alpha$ DMan—green; $\beta$ DGlcA—blue; $\beta$ DXyl—pink; and 6-OAc-red.

Secondly, an orthogonal interaction occurred, which brings the two chains perpendicular to each other. Figure $6 \mathrm{~b}$ shows an example of this. Here chain latching of $\mathrm{cnA}^{\prime}$ occurs in a complementary "gap" region of the $\mathrm{cnD}$ chain where a $\beta \mathrm{DXyl}$ side chain is missing and the mannose backbone is exposed. This allows the $\mathrm{cnA}^{\prime}$ side chains to interact directly with multiple hydrogen bonds to the $\mathrm{cnD}$ mannan backbone. The flexible side chains on either edge of the gap in cnD orient "face-on" to accommodate the $\mathrm{cnA}^{\prime}$ chain. This general mode of interaction is illustrated in the schematic on the right of Figure $6 \mathrm{~b}$, where the $\mathrm{cnA}^{\prime}$ chain is drawn in cross-section.

\section{Discussion}

Our simulations reveal the GXM secondary structure to comprise an extended, relatively inflexible, mannan backbone decorated by hydrophyllic fringes of $\beta$ DGlcA and $\beta$ DXYl side chains 
and a hydrophobic ridge of 6-O-acetyl substitutions. A rigid GXM molecule could help explain some experimental observations from physical chemical studies of GXM and cryptococcal capsules. Probing the polysaccharide capsule with polystyrene beads manipulated with optical tweezers to measure its Young's modulus revealed a relatively stiff structure [28]. The modelling prediction of a linear, inflexible GXM molecule suggests an explanation for the stiffness of the capsule, despite it being composed primarily of water [29]. Further, rigid GXM molecules imply that any increase in molecular mass will translate into molecular length. In this regard, the macroscopic diameter of the capsule size was correlated to the GXM effective diameter measured by light scattering, leading to the proposal that capsule growth is mediated by linear extension of polysaccharide molecules [30]. The prediction that GXM is a rigid molecule is consistent with this observation and suggests how linear growth of individual molecules can translate into increases in capsule size.

Recently, serotype D strains were found to have higher cell surface hydrophobicity than the other serotypes [31]. Although the connection between the predicted molecular structure and cell surface hydrophobicity is not immediately obvious, we note this association and suggest that perhaps the reduced hydrophillic fringes with the concomitant higher exposure of the mannose backbone in $\mathrm{cnD}$ could contribute to this effect.

The GXM secondary structures predicted by this work provide a mechanistic rationale for the importance of 6-O-acetylation for antibody binding: the O-acetyl groups line up in a hydrophobic ridge along the mannan backbone-they are highly exposed for antibody binding and their absence in cnA dramatically alters the exposed binding surface. In addition, O-acetylation reduces the flexibility of the mannan backbone, which may further improve antibody binding be providing a more defined epitope. There is a precedent for this in the Vi capsular polysaccharide of Salmonella typhi which is a linear homopolymer of $\rightarrow 4) \alpha$ DGalNAc $p(1 \rightarrow$ variably O-acetylated at the C-3 position. Immunity of Vi is closely linked to the degree of O-acetylation, a phenomenon which has been attributed to decreased backbone flexibility [32]; modelling has shown O-acetylation to reduce the molecular flexibility of $\mathrm{Vi}$ dramatically [33]. For the GXM mannan backbone, the slight constraint that O-acetylation imposes on every acetylated sugar may be additive in maintaining preferential structures recognised by antibodies.

It may be that protective $\mathrm{mAbs}$ in general bind the O-acetyl ridge (which is more conformationally defined) and that the non-protective Abs bind the far more mobile side chain ridges. For example, the synthetic heptasaccharide (corresponding roughly to an M2 triad) recognised by a number of mAbs (all "non-protective") may induce Ab binding at the side-chain fringe and not the backbone, as the acetylation pattern did not affect binding [21]. Further, Crp127 is an anti-GXM mAb whose binding shows strong serotype dependence, with serotype $\mathrm{D}$ strains bound most strongly, followed by serotype A strains, with little interaction with the C. gattii serotypes B and C [34]. Following our model, it is likely that the $\mathrm{Ab}$ binds primarily to the exposed 6-O-Ac ridge on the backbone, not the fringe regions. The reason for antigenic difference in $C$. gatti is likely the different binding surface exposed: the additional $\beta \mathrm{DXyl}(1 \rightarrow 4)$ side chains in serotypes $\mathrm{B}$ and $\mathrm{C}$ may further hide the mannose backbone and block binding. However, comparative modelling of the $C$. gattii serotypes $B$ and $C$, which feature the $\beta \operatorname{DXyl}(1 \rightarrow 4)$ side chains not investigated in this work, are necessary to confirm this hypothesis.

The GXM structures also provide a mechanistic rationale for why short GXM fragments do not bind to protective antibodies: they are of insufficient length to reproduce the binding surfaces of the GXM polysaccharide: the tetrasaccharide is too short to reproduce the GXM O-acetyl ridge or side chain fringes, which may explain its lack of success as a vaccine candidate. In contrast, the cnAdec' 6-O-acetylates decasaccharide produces a representative segment of the side chain fringes and O-acetyl ridge in the $\mathrm{cnA}^{\prime}$ strand, which explains the success of this epitope in eliciting antibody binding [18]. To reproduce the secondary structure motif in $\mathrm{cnA}^{\prime}$ thus requires a stretch of at least two triads, not the single triad (pentasaccharide) that is suggested by the primary structure. This work thus illustrates the potential utility of molecular modelling in informing the rational design of conjugate vaccines.

Given the inflexibility of the mannan backbone, the role of GXM in the capsule is likely as a scaffold molecule, giving structure and support to the capsule. In addition, this work provides a 
mechanism for self-aggregation of GXM chains [22,24]—chain interlocking occurs spontaneously with complementary stretches on neighbouring GXM strands via strong self-association interactions between mobile side chains and the mannan backbone on the neighbouring strand. We identified two modes of interlocking - a parallel orientation and an orthogonal orientation-both of which could be useful in building a capsule. The chemical structure of GXM predicts linear molecules, but physical chemical studies imply that GXM is a branched structure in solution with properties of a dendrimer [35]. The finding that modelling suggests that GXM molecules have the capacity for self-association provides a mechanism for reconciling molecular linearity with solution branching structures without having to invoke the presence of yet undiscovered chemical structures required for making branched molecules. Consequently, it is possible to envisage how self-associations of individual GXM molecules assemble to form dendrimers by non-covalent interactions.

A function of the side chains could be to facilitate interlocking of the GXM strands: flexible side chains can orient to accommodate and enhance the interlocking interaction. Interaction is likely to be further facilitated in the presence of divalent cations [23] which could pull the chains together. However, as divalent cations cannot be accurately modelled with the standard biomolecular force fields, an alternative simulation methodology will be required to investigate this. Further, varying motifs in a GXM chains may facilitate the random inter-locking of GXM strands: backbone regions with fewer side chains (and hence gaps exposing the mannan backbone) are compatible with regions with increased side chains. For example, if M1 motifs interlock with M2 motifs, then chain interlocking would be facilitated by a serotype D chain (primarily M1) with occasional insertion of M2 motifs. This work therefore also provides a rationale for the unusual heterogeneity in the GXM polysaccharide. There is considerable scope for exploring the mechanisms of self-association with simulations of multiple GXM molecules, and the role of the galactomannan polysaccharide in the capsule, in future work.

\section{Materials and Methods}

To model the GXM polysaccharides, we followed our established systematic molecular modelling approach used in previous work [36-40]. First, we determined the low energy conformations of each of the glycosidic linkages in the polysaccharide by calculation of the $\phi, \psi$ potential of mean force (PMF) for the corresponding disaccharides. We then used these preferred conformations to build reasonable initial conformations for each of the seven oligosaccharides ( $\mathrm{cnX}, \mathrm{cnD}, \mathrm{cnA}, \mathrm{cnA}^{\prime}$, cnAtet, cnAdec and cnAdec') with our CarbBuilder software [41]. These starting structures were then minimised and molecular dynamics simulations run in a water box (with addition of neutralising counter ions when the system contained the charged $\beta$ DGlcA residues). The saccharide conformations were then extracted from the simulation trajectories and analysed to establish the dominant conformations and chain dynamics. Details are as follows.

\subsection{Disaccharide PMF Calculations}

The orientations of the glycosidic linkages in the GXM polysaccharides are each described by two dihedral angles, $\phi$ and $\psi$. For the backbone $\alpha \operatorname{DMan}(1 \rightarrow 3) \alpha$ DMan linkages, these are defined here as $\phi=\mathrm{H} 1-\mathrm{C} 1-\mathrm{O}^{\prime}-\mathrm{C}^{\prime}$ and $\psi=\mathrm{C} 1-\mathrm{O}^{\prime}-\mathrm{C}^{\prime}-\mathrm{H}^{\prime}$. For both the $\beta \mathrm{DGlcA}(1 \rightarrow 2) \alpha \mathrm{DMan}$ the $\beta \mathrm{DXyl}(1 \rightarrow 2) \alpha \mathrm{DMan}$ side chain linkages, $\phi=\mathrm{H} 1-\mathrm{C} 1-\mathrm{O}^{\prime}-\mathrm{C}^{\prime}$ and $\psi=\mathrm{C} 1-\mathrm{O}^{\prime}-\mathrm{C} 2^{\prime}-\mathrm{H} 2^{\prime}$.

We identified the low-energy conformations of the each of the glycosidic linkages in isolation by calculation of the potential of mean force (PMF) for rotation about the $\phi$ and $\psi$ dihedral angles in representative disaccharides; viz., $\alpha \operatorname{DMan}(1 \rightarrow 3) \alpha \operatorname{DMan} ; \beta \operatorname{DGlc}(1 \rightarrow 2) \alpha \mathrm{DMan}$ and $\beta \operatorname{DXyl}(1 \rightarrow 2) \alpha \operatorname{DMan}$ (note that the $\beta \operatorname{DGlcA}(1 \rightarrow 2) \alpha \mathrm{DMan}$ linkage was approximated with the uncharged $\beta$ DGlc $(1 \rightarrow 2) \alpha$ DMan disaccharide). PMFs were calculated with the metadynamics [42] routine incorporated into NAMD [43] using the glycosidic linkage torsion angles as collective variables and the CHARMM36 additive force field for carbohydrates [44,45]. All PMF surfaces were calculated in gas-phase. Gas phase PMFs for uncharged disaccharides have been demonstrated to be a reasonable approximation to solution PMF in a polysaccharide [37,46,47]. Each standard metadynamics simulation 
comprised a $1500 \mathrm{~ns}$ MD simulation, with a Gaussian hill height of 0.5 , hill frequency of 1000 and a hill width of 2.5 degrees. The three PMFs appear in Appendix A, Figure A1 .

\subsection{Molecular Dynamics Simulations}

All simulations were performed with the NAMD molecular dynamics program [43] version 2.12 (employing NAMD CUDA extensions for calculation of long-range electrostatics and nonbonded forces on graphics processing units [48]). Carbohydrates were modelled with the CHARMM36 additive force field for carbohydrates [44,45] and water was simulated with the TIP3P model [49]. Initial configurations of seven oligosaccharides ( $\mathrm{cnX}, \mathrm{cnD}, \mathrm{cnA}, \mathrm{cnA}^{\prime}$, cnAtet, $\mathrm{cnAdec}$ and cnAdec') were built using our CarbBuilder software $[41,50]$ v1.2.23 which employs the psfgen tool to create "protein structure" (psf) files for modelling with the CHARMM force field and the NAMD molecular dynamics program. These initial oligosaccharide structures were optimised with 20,000 steps of standard NAMD minimisation in vacuum and then solvated (using the solvate plugin to the visual molecular dynamics (VMD) [26] analysis package) in a periodic cubic unit cell. The charged saccharides ( $\mathrm{cnD}, \mathrm{cnA}, \mathrm{cnA}^{\prime}$, cnAdec and cnAdec') incorporated randomly distributed sodium ions to electrostatically neutralise the system.

All MD simulations were preceded by a 30,000 step minimisation phase, with a temperature control and equilibration regime involving $10 \mathrm{~K}$ temperature reassignments from $10 \mathrm{~K}$ culminating in a maximum temperature of $300 \mathrm{~K}$. Equations of motion were integrated using a Leap-Frog Verlet integrator with a step size of $1 \mathrm{fs}$ and periodic boundary conditions. Simulations were performed under isothermal-isobaric (nPT) conditions at $300 \mathrm{~K}$ maintained using a Langevin piston barostat [51] and a Nose-Hoover [52,53] thermostat. Long-range electrostatic interactions were treated using particle mesh Ewald (PME) summation, with $\kappa=0.20 \AA^{-1}$ and $1 \AA$ PME grid spacing. Non-bonded interactions were truncated with a switching function applied between 12.0 and $15.0 \AA$ to groups with integer charge. The 1-4 interactions were not scaled, in accordance with the CHARMM force field recommendations. Each system was equilibrated $0.03 \mathrm{~ns}$ with a cycled temperature increase from 0 to $300 \mathrm{~K}$ in $10 \mathrm{~K}$ increments, each cycle commencing with a 10,000 step energy minimisation followed by a $0.001 \mathrm{~ns} \mathrm{MD}$ simulation at the specified temperature until $300 \mathrm{~K}$. For all simulations, structures were collected at intervals of 250 ps for analysis.

\subsubsection{Simulations of Single Saccharide Chains}

The 6-RU strands ( $\mathrm{cnX}, \mathrm{cnD}, \mathrm{cnA}$ and $\mathrm{cnA}^{\prime}$ ) were placed in the centre of a cubic water box with sides of $80 \AA$, while the tetrasaccharide (cnAtet) and decasaccharide (cnAdec and cnAdec') strands employed box lengths of 50 Åand 60 / AA, respectively. The charged systems were neutralised with $\mathrm{Na}^{+}$ions: 6 ions for $\mathrm{cnD}, \mathrm{cnA}$ and $\mathrm{cnA}^{\prime} ; 1$ ion for cnAdec and cnAdec'. All 6-RU MD simulations ran for $500 \mathrm{~ns}$ (with the first $100 \mathrm{~ns}$ treated as equilibration) and the simulations of the shorter fragments (cnAtet, cnAdec and cnAdec') ran for 125 ns (with the first 25 ns treated as equilibration).

\subsubsection{Simulation of Two Saccharide Chains: $\mathrm{cnA}^{\prime}$ and $\mathrm{cnD}$}

For the combined simulation of $\mathrm{cnA}^{\prime}$ and $\mathrm{cnD}, 6-\mathrm{RU}$ strands of each polysaccharide were placed in close proximity within a $80 \AA$ water box with 12 neutralising $\mathrm{Na}^{+}$ions. The combined simulation of $\mathrm{cnA}^{\prime}$ and $\mathrm{cnD}$ ran for $1200 \mathrm{~ns}$, to allow the molecules to relax, diffuse and spontaneously interact.

\subsection{Data Analysis}

Analysis of the simulations used time series frames 25 ps apart. Molecular conformations extracted from the MD simulations were depicted with VMD, where necessary using the PaperChain and Twister visualisation algorithms for carbohydrates [27] to highlight the hexose rings and backbone, respectively. Dihedral angles and end-to-end distances from the simulations were extracted using VMD's Tcl scripting interface and statistical values calculated with in-house Python scripts. Conformations from all MD simulation trajectories were clustered using VMD's internal measure cluster 
command. Clustering analysis used time series frames 250 ps apart, discarding the first $100 \mathrm{~ns}$ as equilibration. First, all simulation conformations were aligned on the two middle mannose residues in the 18 mannose chain (i.e., the ninth and tenth mannose residues). Then all conformations were clustered into families according to a rmsd fit (cutoff of $5 \AA$ ) to all the saccharide rings and glycosidic linkage oxygens in the molecules, ignoring the first two and last two residues in the chain. Only the top four clusters are reported; more minor clusters are ignored.

Author Contributions: Conceptualisation, M.M.K. and S.O.; methodology, M.M.K.; software, M.M.K.; validation, A.C., S.O. and M.M.K.; resources, M.M.K.; data curation, M.M.K.; writing-original draft preparation, M.M.K.; writing-review and editing, A.C., S.O. and M.M.K.; visualisation, M.M.K.; funding acquisition, M.M.K. All authors have read and agreed to the published version of the manuscript.

Funding: This research was funded by THE SOUTH AFRICAN NATIONAL RESEARCH FOUNDATION, grant number 103805.

Acknowledgments: Computations were performed using facilities provided by the University of Cape Town's ICTS High Performance Computing team: http:/ /hpc.uct.ac.za.

Conflicts of Interest: The authors declare no conflict of interest. The funders had no role in the design of the study; in the collection, analyses or interpretation of data; in the writing of the manuscript, or in the decision to publish the results.

\section{Abbreviations}

The following abbreviations are used in this manuscript:

$\mathrm{Ab} \quad$ antibody

cnA 6-RU chains of GXM serogroup A primary motif

$\mathrm{cnA}^{\prime} \quad$ 6-RU chains of GXM serogroup A primary motif, 6-O-acetylated

on every second and third mannose in the RU

cnD 6-RU chains of GXM serogroup D primary motif

cnAtet a tetrasaccharide of GXM serogroup A primary motif

cnAdec a decasaccharide of GXM serogroup A primary motif

cnAdec' a decasaccharide of GXM Serogroup A primary motif 6-O-acetylated on the second and third mannose and last mannose

GXM glucuronoxylomannan polysaccharide

MD molecular dynamics

PMF potential of mean force

RU repeat unit

Appendix A

Appendix A.1
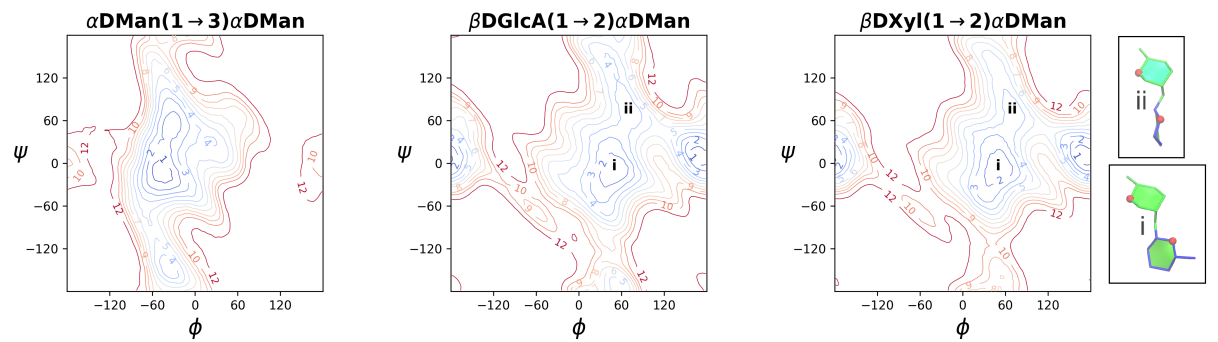

Figure A1. Contoured 2D $\phi, \psi$ PMF surfaces for disaccharide in the gas phase illustrate the range of motion possible for an unrestrained linkage: $\alpha \operatorname{DMan}(1 \rightarrow 3) \alpha \mathrm{DMan}(\mathbf{l e f t}) ; \beta \mathrm{DGlc}(1 \rightarrow 2) \alpha \mathrm{DMan}$ (middle); and $\beta \mathrm{DXyl}(1 \rightarrow 2) \alpha \mathrm{DMan}$ (right). Contours are drawn at intervals of $1 \mathrm{kcal}^{\mathrm{mol}} \mathrm{mo}^{-1}$ to a maximum of 12 kcal.mol ${ }^{-1}$. The $\beta(1 \rightarrow 2)$ PMF maps are annotated with the $\phi, \psi$ regions associated with the (i) major "face-on" and (ii) minor "side-on" linkage orientations. The insets show schematics of the relative rotations of the glycosidic rings in these two orientations. 
Appendix A.2

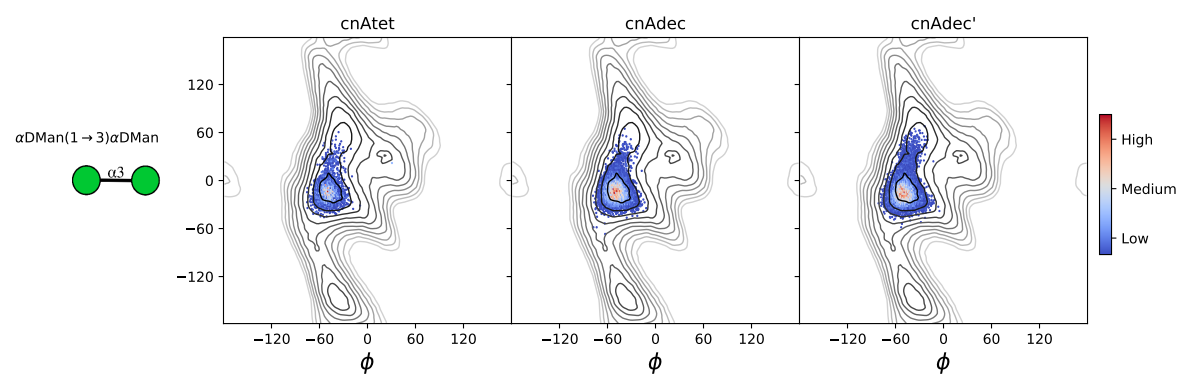

Figure A2. Heat maps of the distribution of the central backbone $\alpha \operatorname{DMan}(1 \rightarrow 3) \alpha \mathrm{DMan}$ glycosidic linkage orientation for a the cnAtet tetrasaccharide fragment (left) and decasaccharide cnAdec (middle) as well as a 6-O-acetylated decasaccharide cnAdec' (right). Glycosidic linkage orientations are shown as rotations of the linkage dihedral angles $\phi$ and $\psi$.

(a)

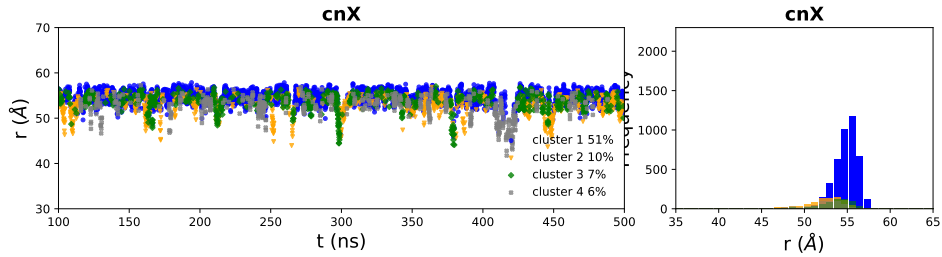

(b)
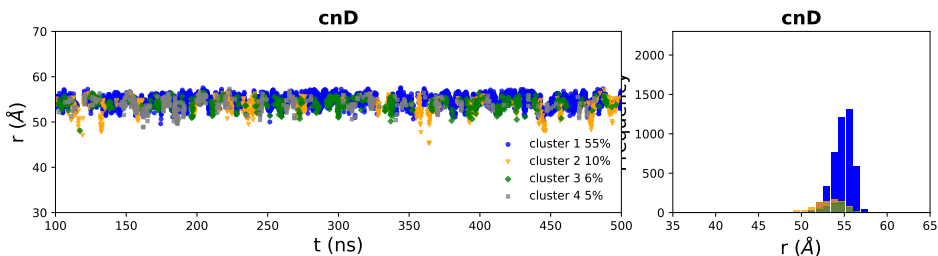

(c)

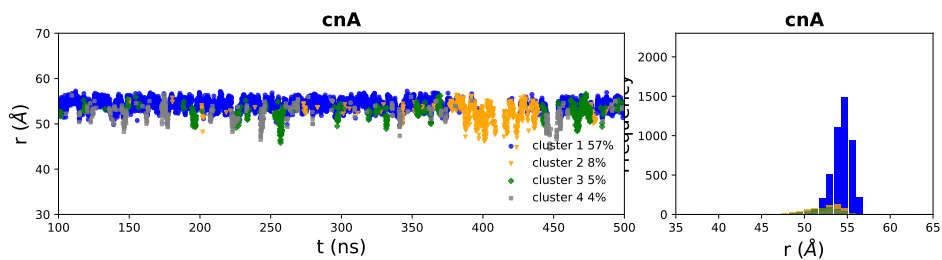

(d)
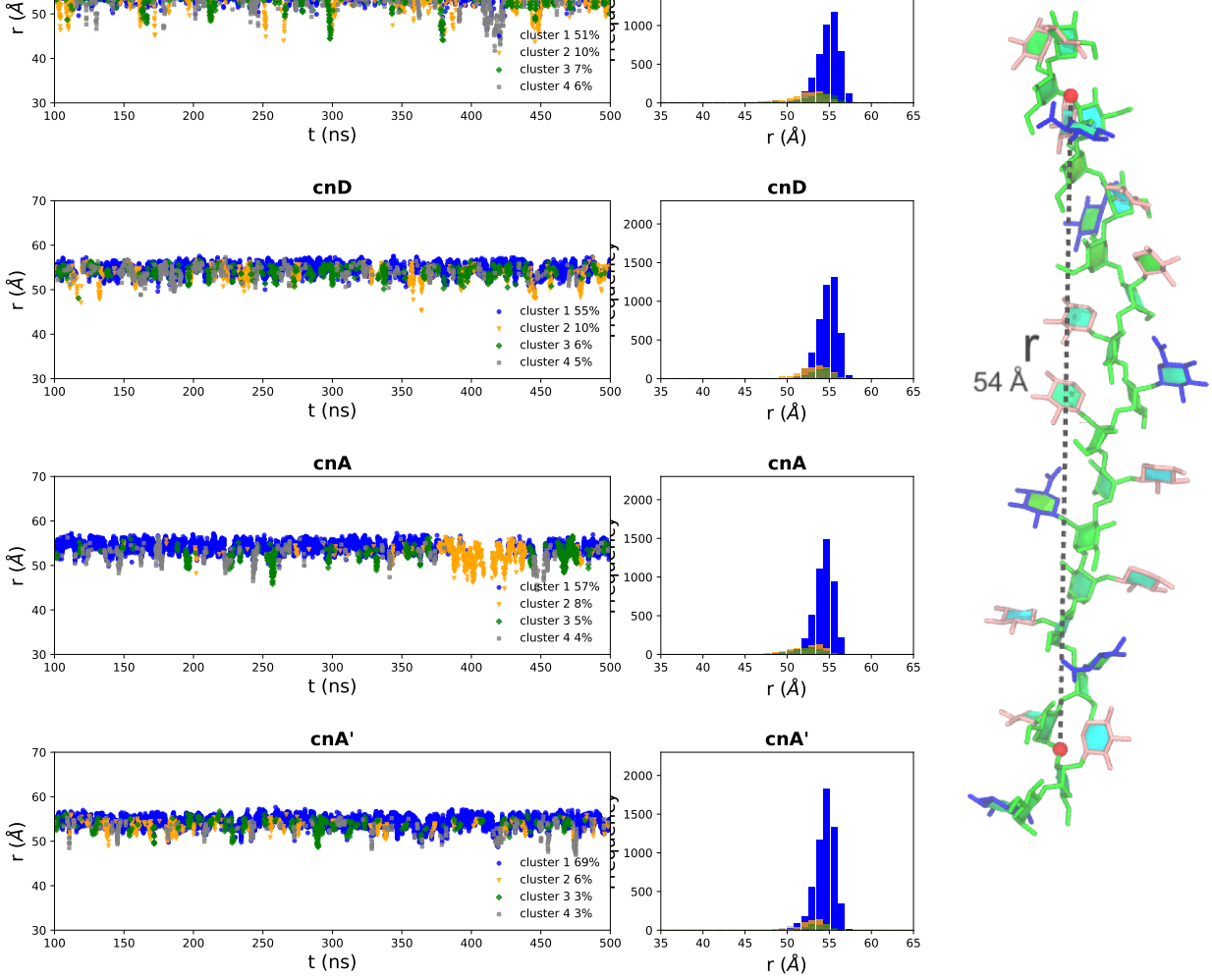

Figure A3. The end-to-end distance, $r$, time series and corresponding histograms of the dominant conformational clusters for the (a) $\mathrm{cnX},(\mathbf{b}) \mathrm{cnD},(\mathbf{c}) \mathrm{cnA}$ and (d) $\mathrm{cnA}^{\prime} \mathrm{GXM}$ chains. For each molecule, the end-to-end distance of the dominant conformational cluster is plotted in blue, the secondary conformational cluster in orange, tertiary in green and quaternary in grey. Lesser clusters are not shown. Percentages for each cluster relative to the total simulation are listed in the legends. The first $100 \mathrm{~ns}$ of simulation are considered equilibration and are not shown. Here $r$ is defined to exclude the two terminal residues on either end of the chain, as the distance from $\mathrm{O} 3$ in the second linkage to $\mathrm{O} 3$ in the 16th linkage in the 18-mannose backbone-labeled for cnA in the image on the right. The residues and substitutions are coloured as follows: $\alpha$ DMan—green; $\beta$ DGlcA—blue; $\beta$ DXyl—pink and 6-OAc-red. 


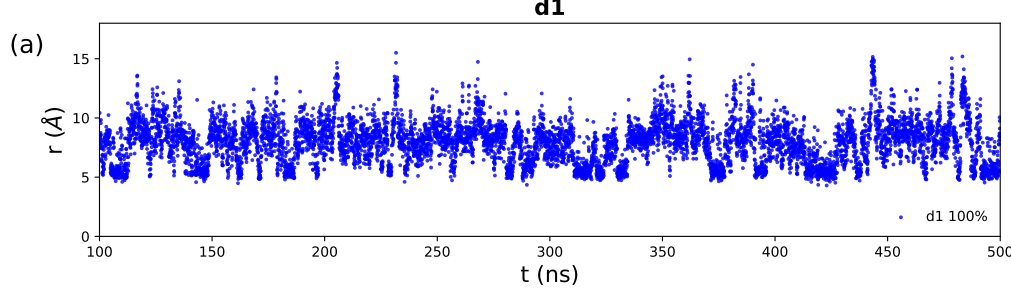

d2

(b)

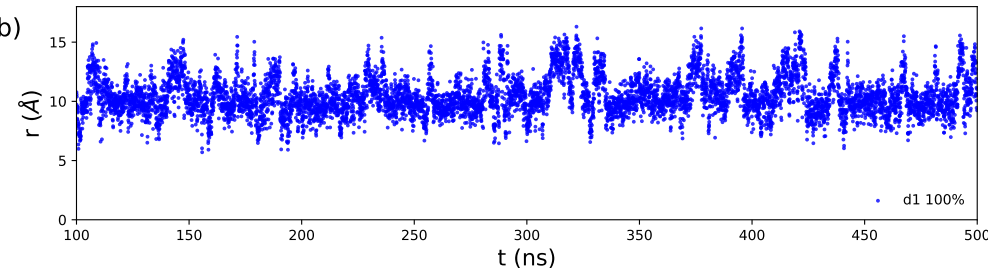

d3

(c)

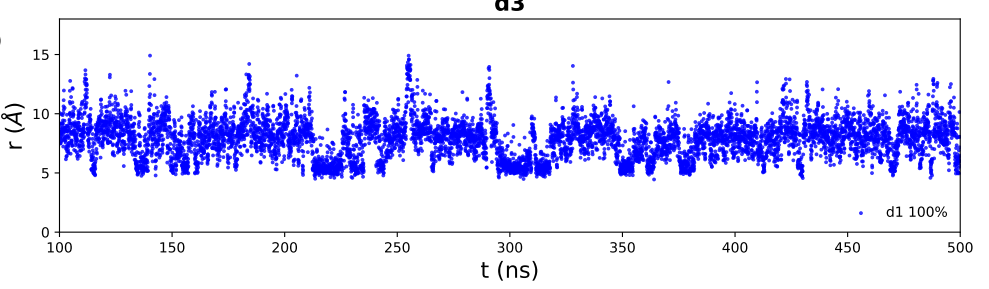

d4

(d)

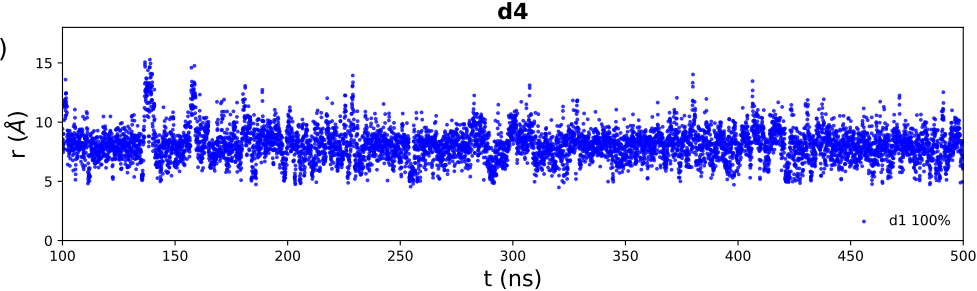

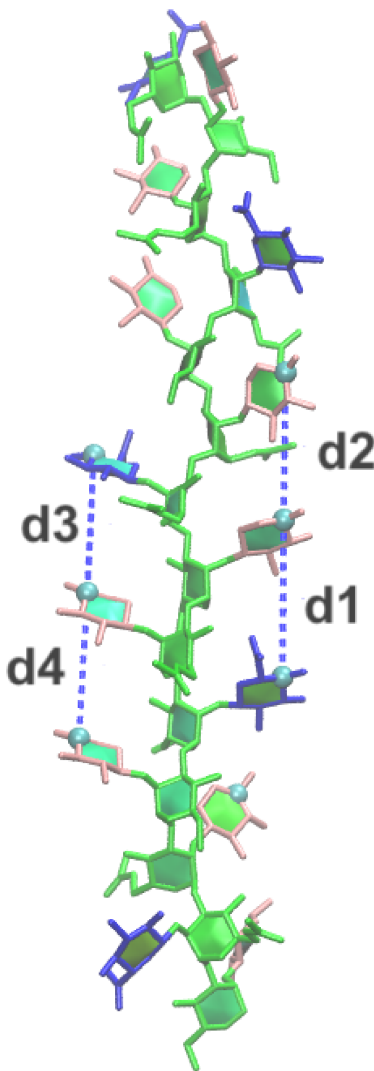

Figure A4. The time series for the distances between interacting side chains in the $\mathrm{cnA}^{\prime}$ molecule labeled on the right. (a) The $\beta$ DGlcA and $\beta$ DXyl distance $\mathrm{d} 1$, (b) the $\beta$ DXyl and $\beta$ DXyl distance $\mathrm{d} 2$ (c) the $\beta \mathrm{DGl} A$ and $\beta \mathrm{DXyl}$ distance $\mathrm{d} 3$ and (d) the $\beta \mathrm{DXyl}$ and $\beta \mathrm{DXyl}$ distance $\mathrm{d} 4$. Here in each case $d$ is defined to as the distance from $\mathrm{C} 4$ in one residue to $\mathrm{C} 4$ in the proximal side chain residue. The first $100 \mathrm{~ns}$ of simulation are considered equilibration and are not shown. The residues and substitutions are coloured as follows: $\alpha$ DMan—green; $\beta$ DGlcA—blue; $\beta$ DXyl—pink and 6-OAc—red.

\section{References}

1. Park, B.J.; Wannemuehler, K.A.; Marston, B.J.; Govender, N.; Pappas, P.G.; Chiller, T.M. Estimation of the current global burden of cryptococcal meningitis among persons living with HIV/AIDS. Aids 2009, 23, 525-530. [CrossRef] [PubMed]

2. McClelland, E.E.; Bernhardt, P.; Casadevall, A. Estimating the relative contributions of virulence factors for pathogenic microbes. Infect. Immun. 2006, 74, 1500-1504. [CrossRef] [PubMed]

3. Zaragoza, O.; Rodrigues, M.L.; Jesus, M.D.; Frases, S.; Dadachova, E.; Casadevall, A. The Capsule of the Fungal Pathogen Cryptococcus neoformans. In Advances in Applied Microbiology; Academic Press: Cambridge, MA, USA, 2009; Volume 68, Chapter 4, pp. 133-216.

4. Kwon-Chung, K.J.; Varma, A. Do major species concepts support one, two or more species within Cryptococcus neoformans? FEMS Yeast Res. 2006, 6, 574-587. [CrossRef] [PubMed]

5. Kwon-Chung, K.J.; Bennett, J.E.; Wickes, B.L.; Meyer, W.; Cuomo, C.A.; Wollenburg, K.R.; Bicanic, T.A.; Castañeda, E.; Chang, Y.C.; Chen, J.; et al. The case for adopting the "species complex" nomenclature for the etiologic agents of cryptococcosis. MSphere 2017, 2, e00357-16. [CrossRef] 
6. Cherniak, R.; Sundstrom, J. Polysaccharide antigens of the capsule of Cryptococcus neoformans. Infect. Immun. 1994, 62, 1507-1512. [CrossRef]

7. Merrifield, E.H.; Stephen, A.M. Structural investigations of two capsular polysaccharides from Cryptococcus neoformans. Carbohydr. Res. 1980, 86, 69-76. [CrossRef]

8. Cherniak, R.; Reiss, E.; Slodki, M.; Plattner, R.; Blumer, S. Structure and antigenic activity of the capsular polysaccharide of Cryptococcus neoformans serotype A. Mol. Immunol. 1980, 17, 1025-1032. [CrossRef]

9. Urai, M.; Kaneko, Y.; Ueno, K.; Okubo, Y.; Aizawa, T.; Fukazawa, H.; Sugita, T.; Ohno, H.; Shibuya, K.; Kinjo, Y.; et al. Evasion of Innate Immune Responses by the Highly Virulent Cryptococcus gattii by Altering Capsule Glucuronoxylomannan Structure. Front. Cell. Infect. Microbiol. 2016, 5, 101. [CrossRef]

10. McFadden, D.C.; Fries, B.C.; Wang, F.; Casadevall, A. Capsule structural heterogeneity and antigenic variation in Cryptococcus neoformans. Eukaryot. Cell 2007, 6, 1464-1473. [CrossRef]

11. Cherniak, R.; Valafar, H.; Morris, L.C.; Valafar, F. Cryptococcus neoformans chemotyping by quantitative analysis of $1 \mathrm{H}$ nuclear magnetic resonance spectra of glucuronoxylomannans with a computer-simulated artificial neural network. Clin. Diagn. Lab. Immunol. 1998, 5, 146-159. [CrossRef]

12. Ellerbroek, P.M.; Lefeber, D.J.; van Veghel, R.; Scharringa, J.; Brouwer, E.; Gerwig, G.J.; Janbon, G.; Hoepelman, A.I.; Coenjaerts, F.E. O-acetylation of cryptococcal capsular glucuronoxylomannan is essential for interference with neutrophil migration. J. Immunol. 2004, 173, 7513-7520. [CrossRef] [PubMed]

13. Varki, A.; Cummings, R.D.; Aebi, M.; Packer, N.H.; Seeberger, P.H.; Esko, J.D.; Stanley, P.; Hart, G.; Darvill, A.; Kinoshita, T.; et al. Symbol Nomenclature for Graphical Representations of Glycans. Glycobiology 2015, 25, 1323-1324. [CrossRef] [PubMed]

14. Neelamegham, S.; Aoki-Kinoshita, K.; Bolton, E.; Frank, M.; Lisacek, F.; Lütteke, T.; O’Boyle, N.; Packer, N.H.; Stanley, P.; Toukach, P.; et al. Updates to the Symbol Nomenclature for Glycans guidelines. Glycobiology 2019, 29, 620-624. [CrossRef] [PubMed]

15. Micoli, F.; Costantino, P.; Adamo, R. Potential targets for next generation antimicrobial glycoconjugate vaccines. FEMS Microbiol. Rev. 2018, 42, 388-423, [CrossRef]

16. Maitta, R.W.; Datta, K.; Chang, Q.; Luo, R.X.; Witover, B.; Subramaniam, K.; Pirofski, L.A. Protective and Nonprotective Human Immunoglobulin M Monoclonal Antibodies to Cryptococcus neoformans Glucuronoxylomannan Manifest Different Specificities and Gene Use Profiles. Infect. Immun. 2004, 72, 4810-4818, doi:10.1128/IAI.72.8.4810-4818.2004. [CrossRef] [PubMed]

17. Nakouzi, A.; Zhang, T.; Oscarson, S.; Casadevall, A. The common Cryptococcus neoformans glucuronoxylomannan M2 motif elicits non-protective antibodies. Vaccine 2009, 27, 3513-3518. [CrossRef]

18. Guazzelli, L.; Ulc, R.; Bowen, A.; Crawford, C.; McCabe, O.; Jedlicka, A.J.; Wear, M.P.; Casadevall, A.; Oscarson, S. A Synthetic Glycan Array Containing Cryptococcus Neoformans Glucuronoxylomannan Capsular Polysaccharide Fragments Allows the Mapping of Protective Epitopes. ChemRxiv 2020. [CrossRef]

19. Casadevall, A.; Mukherjee, J.; Devi, S.J.; Schneerson, R.; Robbins, J.B.; Scharff, M.D. Antibodies elicited by a Cryptococcus neoformans-tetanus toxoid conjugate vaccine have the same specificity as those elicited in infection. J. Infect. Dis. 1992, 165, 1086-1093. [CrossRef]

20. Casadevall, A.; Coelho, C.; Cordero, R.J.; Dragotakes, Q.; Jung, E.; Vij, R.; Wear, M.P. The capsule of Cryptococcus neoformans. Virulence 2019, 10, 822-831. [CrossRef]

21. Oscarson, S.; Alpe, M.; Svahnberg, P.; Nakouzi, A.; Casadevall, A. Synthesis and immunological studies of glycoconjugates of Cryptococcus neoformans capsular glucuronoxylomannan oligosaccharide structures. Vaccine 2005, 23, 3961-3972. [CrossRef]

22. McFadden, D.C.; De Jesus, M.; Casadevall, A. The physical properties of the capsular polysaccharides from Cryptococcus neoformans suggest features for capsule construction. J. Biol. Chem. 2006, 281, 1868-1875. [CrossRef] [PubMed]

23. Nimrichter, L.; Frases, S.; Cinelli, L.P.; Viana, N.B.; Nakouzi, A.; Travassos, L.R.; Casadevall, A.; Rodrigues, M.L. Self-aggregation of Cryptococcus neoformans capsular glucuronoxylomannan is dependent on divalent cations. Eukaryot. Cell 2007, 6, 1400-1410. [CrossRef]

24. Frases, S.; Nimrichter, L.; Viana, N.B.; Nakouzi, A.; Casadevall, A. Cryptococcus neoformans capsular polysaccharide and exopolysaccharide fractions manifest physical, chemical, and antigenic differences. Eukaryot. Cell 2008, 7, 319-327. [CrossRef] [PubMed] 
25. Kuttel, M.M.; Ravenscroft, N. The Role of Molecular Modeling in Predicting Carbohydrate Antigen Conformation and Understanding Vaccine Immunogenicity. In Carbohydrate-Based Vaccines: From Concept to Clinic; ACS Symposium Series; American Chemical Society: Washington, DC, USA, 2018; Volume 1290, Chapter 7, pp. 139-173.

26. Humphrey, W.; Dalke, A.; Schulten, K. VMD—Visual Molecular Dynamics. J. Mol. Graph. 1996, 14, $33-38$. [CrossRef]

27. Cross, S.; Kuttel, M.M.; Stone, J.E.; Gain, J.E. Visualisation of cyclic and multi-branched molecules with VMD. J. Mol. Graph. Modell. 2009, 28, 131-139. [CrossRef]

28. Frases, S.; Pontes, B.; Nimrichter, L.; Rodrigues, M.L.; Viana, N.B.; Casadevall, A. The elastic properties of the Cryptococcus neoformans capsule. Biophys. J. 2009, 97, 937-945. [CrossRef] [PubMed]

29. Maxson, M.E.; Cook, E.; Casadevall, A.; Zaragoza, O. The volume and hydration of the Cryptococcus neoformans polysaccharide capsule. Fungal Genet. Biol. 2007, 44, 180-186. [CrossRef]

30. Frases, S.; Pontes, B.; Nimrichter, L.; Viana, N.B.; Rodrigues, M.L.; Casadevall, A. Capsule of Cryptococcus neoformans grows by enlargement of polysaccharide molecules. Proc. Natl. Acad. Sci. USA 2009, 106, 1228-1233. [CrossRef]

31. Vij, R.; Crawford, C.J.; Casadevall, A. Variation in cell surface hydrophobicity among Cryptococcus neoformans strains influences interactions with amoeba. mSphere 2020, 5, e00310-20.[CrossRef]

32. Szu, S.C.; Li, X.; Stone, A.L.; Robbins, J.B. Relation between structure and immunologic properties of the Vi capsular polysaccharide. Infect. Immun. 1991, 59, 4555-4561. [CrossRef]

33. Hitri, K.; Kuttel, M.M.; De Benedetto, G.; Lockyer, K.; Gao, F.; Hansal, P.; Rudd, T.R.; Beamish, E.; Rijpkema, S.; Ravenscroft, N.; et al. O-acetylation of typhoid capsular polysaccharide confers polysaccharide rigidity and immunodominance by masking additional epitopes. Vaccine 2019, 37, 3866-3875. [CrossRef] [PubMed]

34. Probert, M.; Zhou, X.; Goodall, M.; Johnston, S.A.; Bielska, E.; Ballou, E.R.; May, R.C. A glucuronoxylomannan epitope exhibits serotype-specific accessibility and redistributes towards the capsule surface during titanization of the fungal pathogen Cryptococcus neoformans. Infect. Immun. 2019, 87, e00731-18. [CrossRef] [PubMed]

35. Cordero, R.J.; Frases, S.; Guimaräes, A.J.; Rivera, J.; Casadevall, A. Evidence for branching in cryptococcal capsular polysaccharides and consequences on its biological activity. Mol. Microbiol. 2011, 79, 1101-1117. [CrossRef] [PubMed]

36. Kuttel, M.M.; Jackson, G.E.; Mafata, M.; Ravenscroft, N. Capsular polysaccharide conformations in pneumococcal serotypes 19F and 19A. Carbohydr. Res. 2015, 406, 27-33. [CrossRef]

37. Kuttel, M.M.; Timol, Z.; Ravenscroft, N. Cross-protection in Neisseria meningitidis serogroups Y and W polysaccharides: A comparative conformational analysis. Carbohydr. Res. 2017, 446-447, 40-47. [CrossRef] [PubMed]

38. Hlozek, J.; Kuttel, M.M.; Ravenscroft, N. Conformations of Neisseria meningitidis serogroup A and X polysaccharides: The effects of chain length and O-acetylation. Carbohyd. Res. 2018, 465, 44-51. [CrossRef] [PubMed]

39. Kuttel, M.M.; Ravenscroft, N. Conformation and cross-protection in Group B Streptococcus serotype III and Streptococcus pneumoniae serotype 14: A molecular modeling study. Pharmaceuticals 2019, 12, 28. [CrossRef]

40. Hlozek, J.; Ravenscroft, N.; Kuttel, M.M. The Effects of Glucosylation and O-Acetylation on the Conformation of Shigella Flexneri Serogroup 2 O-Antigen Vaccine Targets. J. Phys. Chem. B 2020, 124, 2806-2814. [CrossRef]

41. Kuttel, M.M.; Stähle, J.; Widmalm, G. CarbBuilder: Software for Building Molecular Models of Complex Oligo- and Polysaccharide Structures. J. Comput. Chem. 2016, 37, 2098-2105. [CrossRef]

42. Laio, A.; Parrinello, M. Escaping free energy minima. Proc. Natl. Acad. Sci. USA 2002, 99, 12562-12565. [CrossRef]

43. Phillips, J.C.; Braun, R.; Wang, W.; Gumbart, J.; Tajkhorshid, E.; Villa, E.; Chipot, C.; Skeel, R.D.; Kale, L.; Schulten, K. Scalable Molecular Dynamics with NAMD. J. Comput. Chem. 2005, 26, 1781-1802. [CrossRef] [PubMed]

44. Guvench, O.; Greene, S.N.; Kamath, G.; Brady, J.W.; Venable, R.M.; Pastor, R.W.; MacKerell, A.D. Additive Empirical Force Field for Hexopyranose Monosaccharides. J. Comput. Chem. 2008, 29, 2543-2564. [CrossRef] [PubMed] 
45. Guvench, O.; Hatcher, E.; Venable, R.M.; Pastor, R.W.; Alexander, D.; MacKerell, J. CHARMM Additive All-Atom Force Field for Glycosidic Linkages between Hexopyranoses. J. Chem. Theory Comput. 2009, 5, 2353-2370. [CrossRef] [PubMed]

46. Yang, M.; MacKerell, A.D. Conformational Sampling of Oligosaccharides Using Hamiltonian Replica Exchange with Two-Dimensional Dihedral Biasing Potentials and the Weighted Histogram Analysis Method (WHAM). J. Chem. Theory Comput. 2015, 11, 788-799. [CrossRef]

47. Kuttel, M.M. Conformational free energy maps for globobiose ( $\alpha$-D-Gal-(1-4)- $\beta$-D-Gal) in implicit and explict aqueous solution. Carbohydr. Res. 2008, 343, 1091-1098. [CrossRef]

48. Stone, J.E.; Phillips, J.C.; Freddolino, P.L.; Hardy, J.; Trabuco, L.G.; Schulten, K. Accelerating Molecular Modeling Applications with Graphics Processors. J. Comput. Chem. 2007, 28, 2618-2639. [CrossRef]

49. Jorgensen, W.L.; Chandrasekhar, J.; Madura, J.D.; Impey, R.W.; Klein, M.L. Comparison of simple potential functions for simulations of liquid water. J. Chem. Phys. 1983, 79, 926-935. [CrossRef]

50. Kuttel, M.; Ravenscroft, N.; Foschiatti, M.; Cescutti, P.; Rizzo, R. Conformational properties of two exopolysaccharides produced by Inquilinus limosus, a cystic fibrosis lung pathogen. Carbohydr. Res. 2012, 350, 40-48. [CrossRef]

51. Feller, S.E.; Zhang, Y.; Pastor, R.W.; Brooks, B.R. Constant pressure molecular dynamics simulation: The Langevin piston method. J. Chem. Phys. 1995, 103, 4613-4621. [CrossRef]

52. Nose, S.; Lein, M.L. Constant pressure molecular dynamics for molecular systems. Mol. Phys. 1983, 50, $1055-1076$. [CrossRef]

53. Hoover, W.G. Canonical dynamics: Equilibrium phase-space distributions. Phys. Rev. A 1985, 31, $1965-1697$. [CrossRef] [PubMed]

(C) 2020 by the authors. Licensee MDPI, Basel, Switzerland. This article is an open access article distributed under the terms and conditions of the Creative Commons Attribution (CC BY) license (http:/ / creativecommons.org/licenses/by/4.0/). 